\title{
Structure Specific Liquid Crystal Anchoring Induced by the Molecular Combing of Short Oligonucleotides
}

\section{Supporting Information}

Saonti Chakraborty, Patrick S. Noonan, Jon Monserud, Daniel K Schwartz*

Department of Chemical and Biological Engineering

University of Colorado Boulder, Boulder, CO 80309

*To whom correspondence should be addressed: daniel.schwartz@colorado.edu 


\section{Table of Contents}

\begin{tabular}{|c|c|}
\hline TIRF-Microscopy Image of Surface Immobilized DNA On GPTMS & S-3 \\
\hline Polarized light images of hybrid aligned LC cells containing DNA & S-5 - S-8 \\
\hline Sample orientation used to determine the LC alignment & S-5 \\
\hline $\begin{array}{l}\text { Polarized-Microscopy Images for hybridized and unhybridized } \\
\text { DNA }\end{array}$ & S-5 - S-6 \\
\hline $\begin{array}{l}\text { Polarized-Microscopy Images for varying "Probe" DNA lengths } \\
\text { Distribution of dark pixels for varying "Probe" DNA lengths }\end{array}$ & S-8 \\
\hline
\end{tabular}




\section{TIRF-Microscopy (TIRFM) Image of Surface Immobilized DNA on GPTMS}

TIRFM was used to measure the surface density of immobilized probe ssDNA. For surface density experiments, serial dilutions of amine modified DNA labeled with Alexa Flour 488 were prepared ranging from $100 \mathrm{nM}-100 \mathrm{pM}$. GPTMS surfaces were prepared and incubated in each dilution.

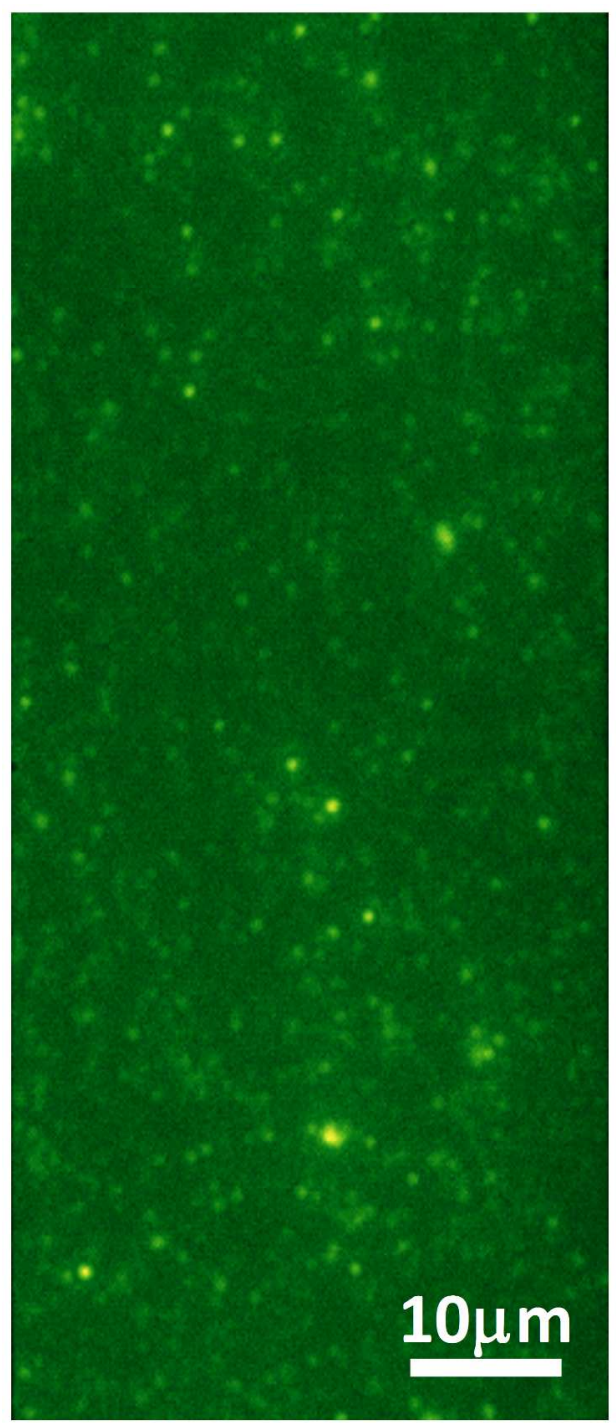

Figure S1. Example TIRF microscopy image of GPTMS surface with covalently attached AlexaFluor 488 modied DNA. 
After rinsing and hot water bath treatment, the samples were examined with TIRFM; the immobilized ssDNA was directly excited with a $491 \mathrm{~nm}$ wavelength laser. Images were obtained with $1 \mathrm{~s}$ acquisition times, individual ssDNA molecules were identified as diffraction limited objects. The number of immobilized ssDNA molecules was counted, and the image intensity was integrated to get the total intensity of each image. Over twenty locations were examined on each sample and experiments were run in triplicate for each dilution. Using the collected data, a calibration plot of intensity versus surface density was prepared. At greater surface densities of ssDNA it became impossible to identify individual molecules. Therefore, assuming that linearity was retained, the calibration plot was extrapolated to higher total intensities to extract an estimated surface density of ssDNA $^{1}$. For samples prepared using the $100 \mathrm{nM}$ ssDNA concentration the grafting density of ssDNA was estimated to be $\sim 14$ oligos $/ \mu \mathrm{m}^{2}$.

\section{Polarized light images of hybrid aligned LC cells containing DNA}

Sample orientation used to determine the LC alignment - 


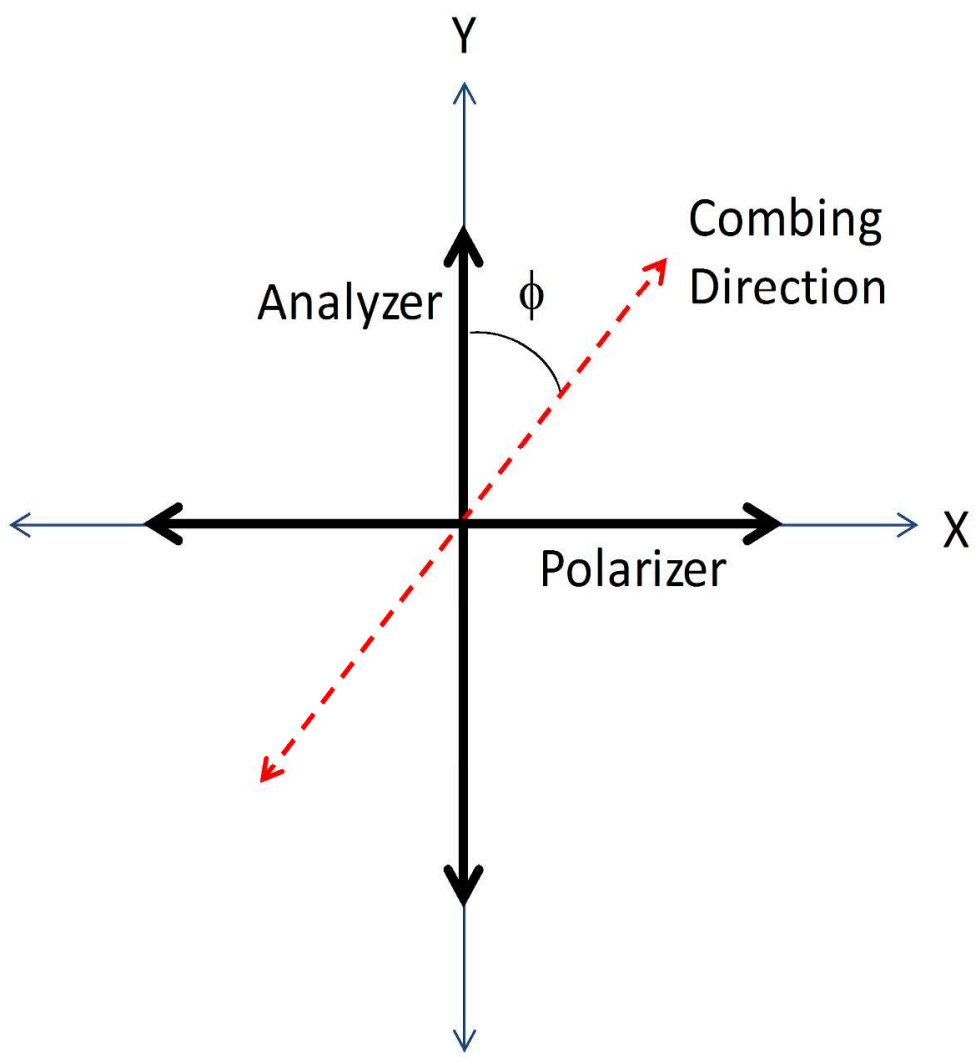

Figure S2. Sample orientation used to determine the LC alignment. The crossed analyzer and polarizer, respectively. " $\phi$ " is the angle between the combing direction (i.e., the extension direction of DNA) and the analyzer.

\section{Polarized-Microscopy Images for hybridized and unhybridized DNA-}

The polarized images shown in figures S3 and S4 were obtained on hybrid LC cell, that were made of DNA decorated GPTMS surface and a substrate with OTES monolayer that induced homeotropoic orientation at the opposing interface. The series of images is shown to elucidate the variation of transmitted intensity through the samples, thereby, giving an estimate to angle of extinction. Images at $0^{\circ}$ and $30^{\circ}$ of Figure $\mathrm{S} 3$ are already shown in the manuscript (Figure $3(\mathrm{a})$ ). 


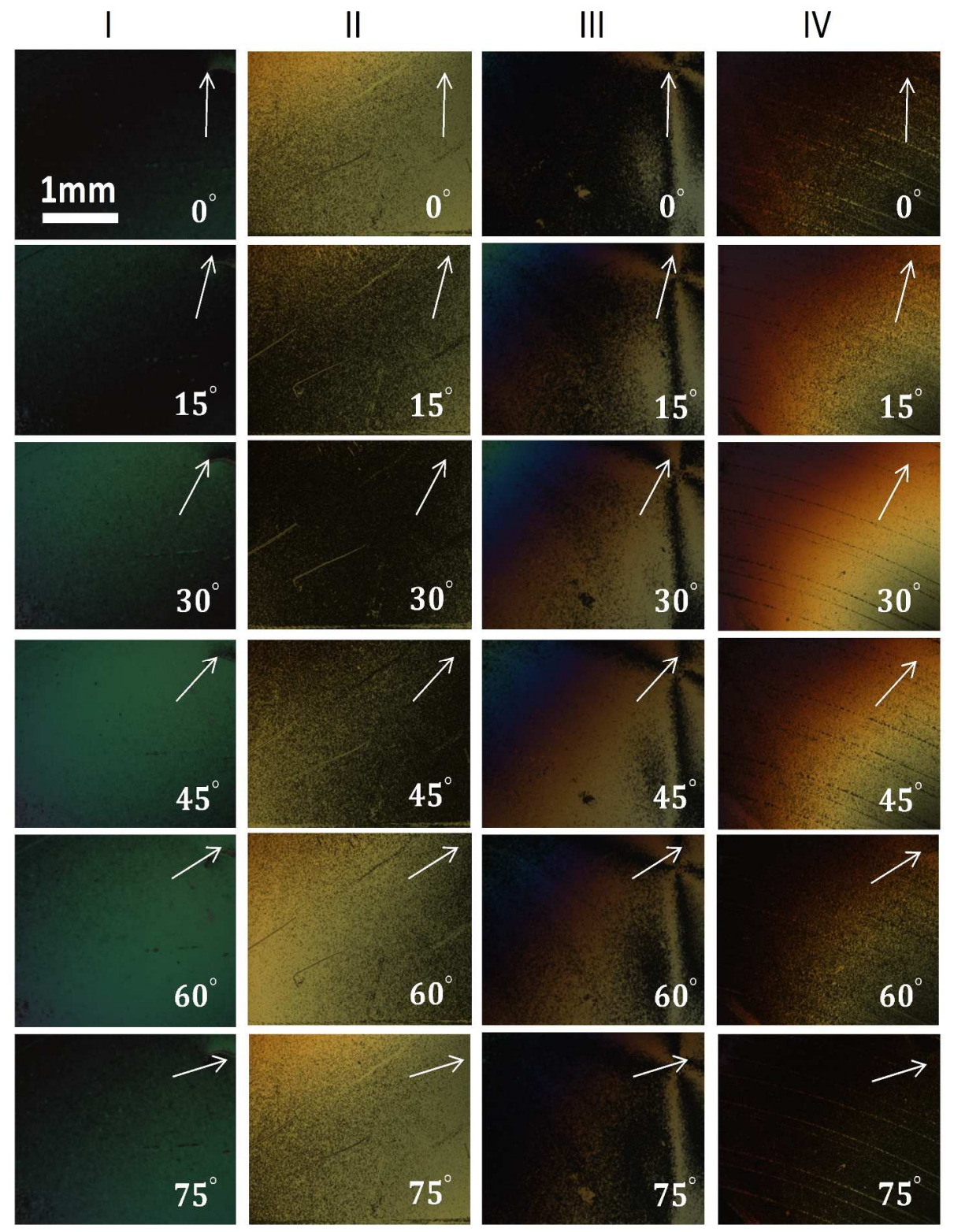

Figure S3. Polarized light microscopy images of the hybrid LC cells made with GPTMS and OTES monolayers containing (I) ssDNA, (II) dsDNA, (III) ssDNA and non-complimentary DNA and (IV) ssDNA and mismatch DNA. The figure shows images that were obtained by varying the angle $(\phi)$ between the combing direction and crossed polarizers from $0^{\circ}$ to $90^{\circ}$. The figure contains images that are already shown in Figure 2(a) of the manuscript along with the additional images. The angles $(\phi)$ are written on the respective images while the white arrows indicate the direction of DNA combing. 


\section{Polarized-Microscopy Images for varying "Probe" DNA lengths -}

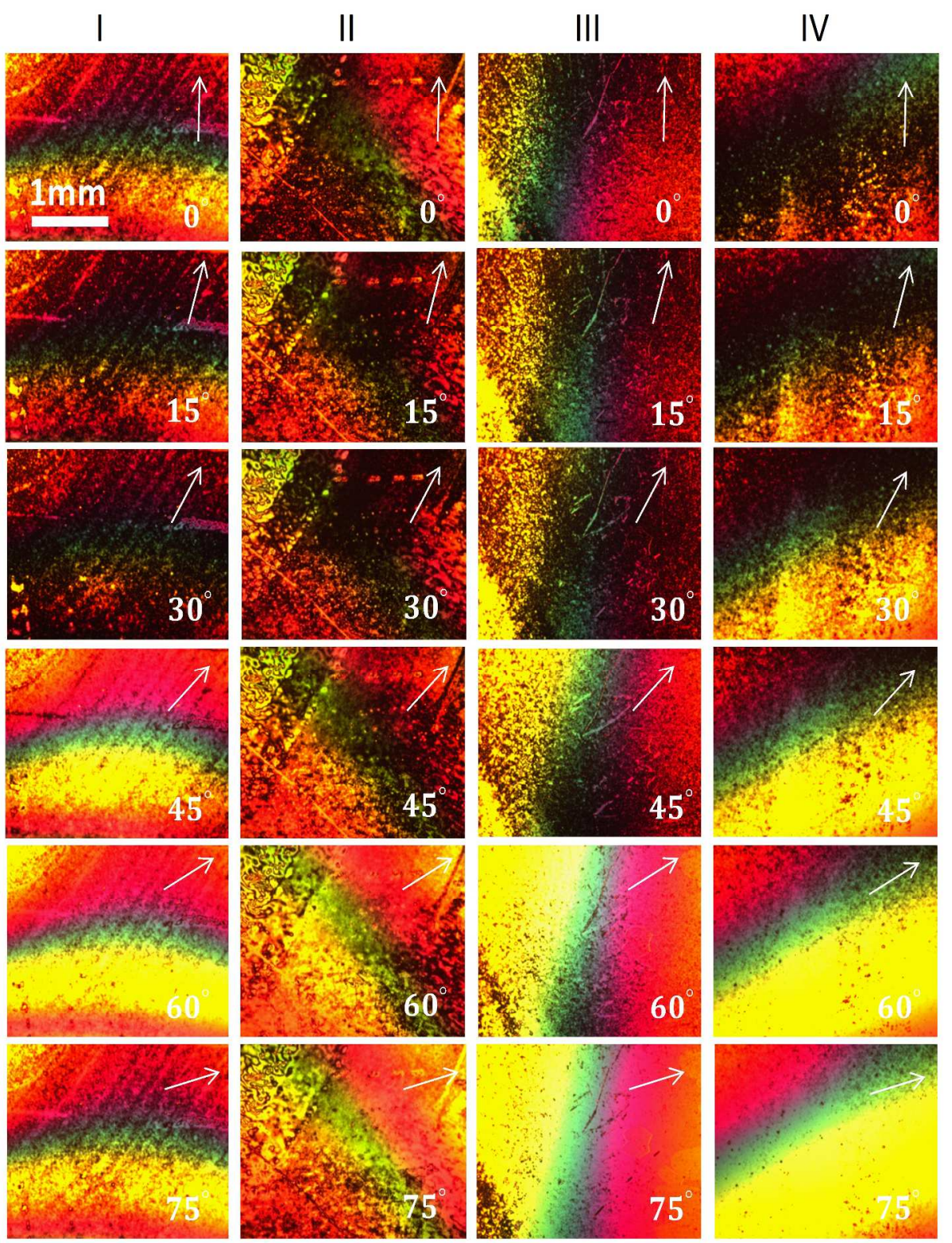

Figure S4. Polarized light microscopy images of the hybrid LC cells made with GPTMS and OTES monolayers containing (I) $\mathrm{F}_{\mathrm{ss}}=0.2$, (II) $\mathrm{F}_{\mathrm{ss}}=0.33$, (III) $\mathrm{F}_{\mathrm{ss}}=0.5$ and (IV) $\mathrm{F}_{\mathrm{ss}}=0.67$. The figure shows images that were obtained by varying the angle $(\phi)$ between the combing direction and crossed polarizers from $0^{\circ}$ to $90^{\circ}$. The angles are written on the respective images while the white arrows indicate the direction of DNA combing. 
The histograms shown below represent the ratio of number dark pixels for a given angle between the stretching direction and analyzer $(\phi)$ and the total number of pixels in the image.

(a)

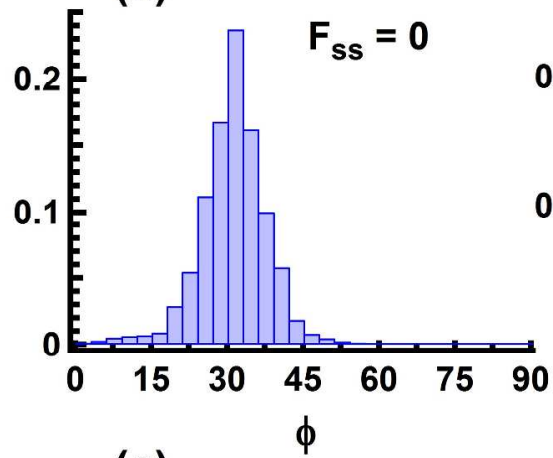

(b)

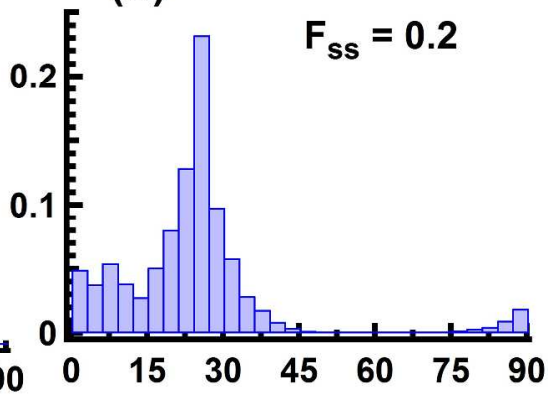

(d)

$\phi$

$F_{\mathrm{ss}}=0.5$
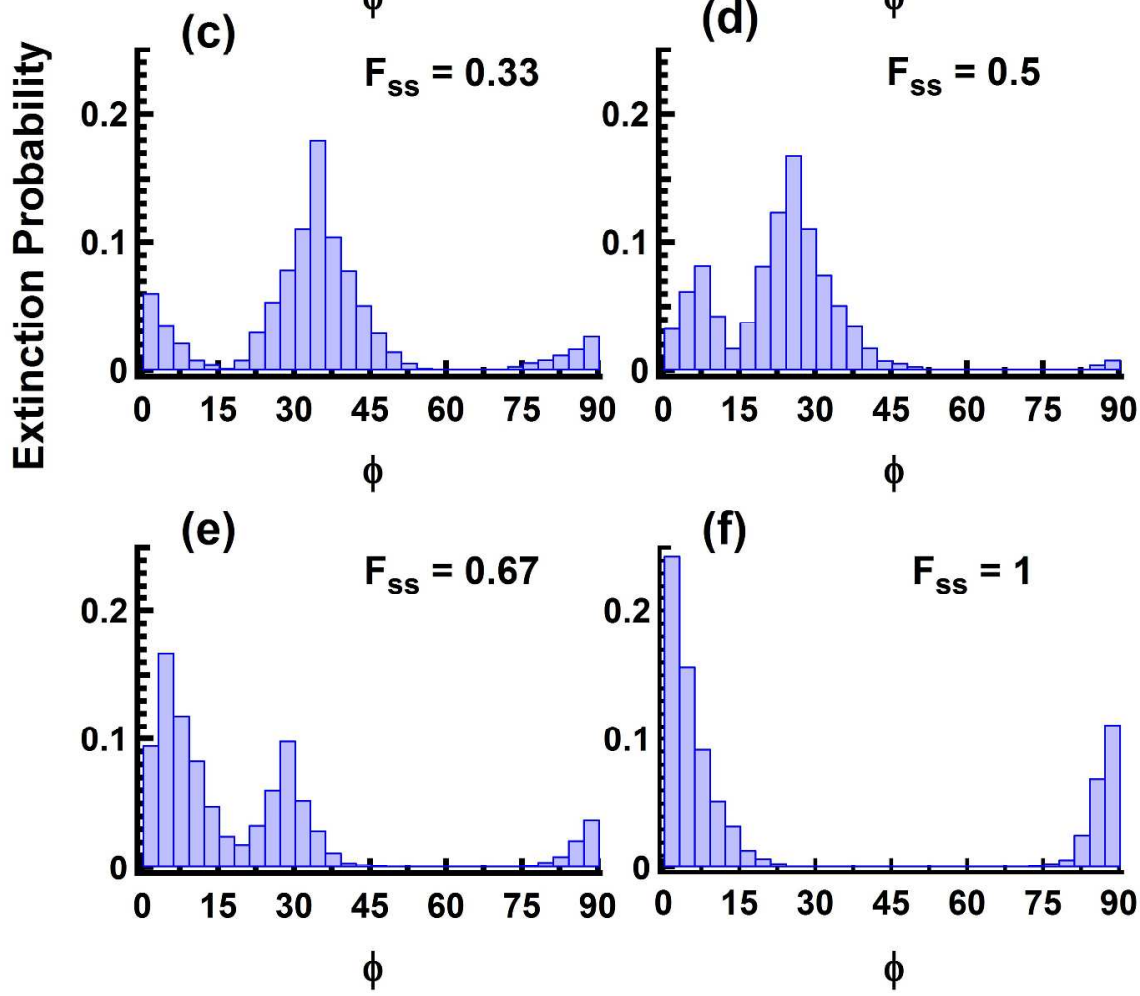

Figure S5. Histogram showing the distribution of dark pixels at every angle for (a) pure $\operatorname{dsDNA}\left(\mathrm{F}_{\mathrm{ss}}=0\right),(\mathrm{b}) \mathrm{F}_{\mathrm{ss}}=0.2$, (c) $\mathrm{F}_{\mathrm{ss}}=0.33$, (d) $\mathrm{F}_{\mathrm{ss}}=0.5$, (e) $\mathrm{F}_{\mathrm{ss}}=0.67$ and (f) pure ssDNA $\left(\mathrm{F}_{\mathrm{ss}}=1\right)$. Histograms (a) and (e) are already shown in Figure 4 (a) and (b) of the manuscript.

\section{References}

1. Monserud, J. H.; Schwartz, D. K. Mechanisms of Surface-Mediated DNA Hybridization. Acs Nano 2014, 8 (5), 4488-4499. 